\title{
Attenuation of Alfvén waves in straight and curved coronal slabs
}

\author{
M. Gruszecki ${ }^{1}$, K. Murawski ${ }^{1}$, S. K. Solanki ${ }^{2}$, and L. Ofman ${ }^{3}$ \\ ${ }^{1}$ Group of Astrophysics and Gravity Theory, Institute of Physics, UMCS, ul. Radziszewskiego 10, 20-031 Lublin, Poland \\ e-mail: marcingruszecki@wp.pl \\ 2 Max-Planck-Institut für Sonnensystemforschung, Max-Planck-Str. 2, 37191 Katlenburg-Lindau, Germany \\ 3 The Catholic University of America, NASA Goddard Space Flight Center, Code 671, Greenbelt, MD 20771, USA
}

Received 12 December 2006 / Accepted 28 March 2007

\begin{abstract}
Aims. We consider impulsively generated Alfvén waves in coronal loops to investigate the role of energy leakage on wave attenuation, which includes lateral leakage, leakage into dense photospheric regions and nonlinear driving of magnetosonic waves.

Methods. A coronal loop is modelled either as a straight magnetic slab or as a curved slab of smooth mass density profiles. We perform numerical simulations of 2.5D ideal magnetohydrodynamic equations to determine the signatures of Alfvén waves.

Results. The numerical results show that lateral leakage of Alfvén waves is significant in comparison to leakage into the photospheric regions for realistic corona to photospheric density ratios. Energy leakage is enhanced by curvature of magnetic field lines and for large amplitude Alfvén waves for which nonlinear driving of magnetosonic waves is more significant than in the linear regime.
\end{abstract}

Key words. magnetohydrodynamics (MHD) - Sun: corona - Sun: oscillations

\section{Introduction}

The good spatial resolution of highly sensitive instruments such as SUMER (SOHO) and TRACE resulted in the detection of various oscillation modes in solar coronal loops. The observed oscillations include propagating (De Moortel et al. 2002) and standing (Wang et al. 2002) slow magnetosonic waves. There are also observations of a branch of fast magnetosonic waves: horizontal kink (Aschwanden et al. 1999; Nakariakov et al. 1999; Schrijver et al. 2002; Aschwanden et al. 2002), vertical kink (Wang \& Solanki 2004), and fast sausage (Pascoe et al. 2007). It has been suggested that velocity fluctuations that are associated with torsional Alfvén waves can be observed through variations of the Doppler shifts (e.g., Zaqarashvili 2003), although a direct detection still has not been made.

Magnetic loop oscillations were studied analytically over the last few decades by Edwin \& Roberts (1982, 1983), Roberts et al. (1984), Cally (1999), Nakariakov (2003), Ofman (2002), Van Doorsselaere et al. (2004). As analytical studies are amenable only to highly idealized situations, numerical simulations are often required to tackle more complex models. Such a numerical approach was undertaken by a number of authors. For instance, Mendoza-Briceno et al. (2004), Nakariakov et al. (2004) and Selwa et al. (2005) studied slow modes in a onedimensional magnetic field. More complex models were developed for fast magnetosonic oscillations. For instance, in their early studies, Murawski \& Roberts (1994) considered impulsively generated linear and nonlinear fast magnetosonic waves in a two-dimensional coronal slab. Selwa et al. (2004) performed numerical simulations of propagating slow, fast sausage, and fast kink waves in a three-dimensional geometry. Ofman (2002) studied leakage of Alfvén waves from coronal loops into the chromosphere using a 1.5D MHD model. He found that the leakage time of long-wavelength oscillations is five times longer than that obtained from observational data recorded by the TRACE satellite (e.g., Del Zanna et al. 2005). Attenuation of kink oscillations by energy leakage and resonant absorption in curved coronal loops was discussed recently by Terradas et al. (2006). Various wave damping mechanisms were reviewed by Roberts (2000). The mechanisms include resonance absorption, non-ideal effects, loop curvature, and phase mixing.

Despite significant achievements in the development of realistic models, there are still considerable gaps in our understanding of wave phenomena in coronal loops. A goal of this paper is to extend the $1.5 \mathrm{D}$ model of Ofman (2002) into its $2.5 \mathrm{D}$ ana$\log$ in which straight and curved plasma slabs are considered. Our work also aims towards obtaining a better insight into the observed transverse loop oscillations (Aschwanden et al. 1999; Nakariakov et al. 1999; Schrijver et al. 2002; Aschwanden et al. 2002), and the possible mechanisms of their damping. This paper is organized as follows. Numerical models are described in the following section. Numerical results are presented and discussed in Sect. 3. This paper is concluded by a summary of the main results in Sect. 4.

\section{Numerical models}

In our models we neglect non-ideal effects and the gravity force. To describe coronal plasma we use the ideal magnetohydrodynamic (MHD) equations:

$\frac{\partial \varrho}{\partial t}+\nabla \cdot(\varrho \boldsymbol{V})=0$

$\varrho \frac{\partial \boldsymbol{V}}{\partial t}+\varrho(\boldsymbol{V} \cdot \nabla) \boldsymbol{V}=-\nabla p+\frac{1}{\mu}(\nabla \times \boldsymbol{B}) \times \boldsymbol{B}$,

$\frac{\partial \boldsymbol{B}}{\partial t}=\nabla \times(\boldsymbol{V} \times \boldsymbol{B})$

$\frac{\partial p}{\partial t}+\nabla \cdot(p \boldsymbol{V})=(1-\gamma) p \nabla \cdot \boldsymbol{V}$,

$\nabla \cdot \boldsymbol{B}=0$. 
Here $\varrho$ is mass density, $p$ is gas pressure, $\boldsymbol{B}$ is magnetic field, $\boldsymbol{V}=\left[V_{x}, V_{y}, V_{z}\right]$ is flow velocity, $\mu$ is the magnetic permeability and $\gamma=5 / 3$ is the adiabatic index. We limit our discussion to a $2.5 \mathrm{D}$ magnetically structured medium in which the equilibrium magnetic field is polarized in the $x-z$ plane and all plasma variables are invariant in the $y$-direction, $\partial / \partial y=0$, but perturbed velocity and magnetic field $y$-components are not identical to zero. In such an idealized medium $V_{x}$ and $V_{z}$ correspond to magnetosonic waves which are coupled. The $y$-component of velocity, $V_{y}$, is associated with the Alfvén wave which decouples from the magnetosonic waves. However, the Alfvén wave is able to drive the magnetoacoustic waves through nonlinear terms (e.g., Murawski 1992; Nakariakov et al. 1997). Driven in this way, magnetosonic waves do not react back on the Alfvén wave, and there is no resonant absorption.

\subsection{A straight slab model}

We consider an equilibrium which corresponds to a simple straight slab model. For a straight slab the magnetic field can be chosen to be uniform over the whole space and directed along the $x$-direction, $\boldsymbol{B}_{0}=B_{0} \hat{\boldsymbol{x}}$. The equilibrium plasma quantities represent an enhanced mass density over a width $2 a$ in an otherwise uniform medium, viz.

$p(z), \varrho(z), B(z)=\left\{\begin{array}{l}p_{0}, \varrho_{\mathrm{i}}, B_{0},|z| \leq a, \\ p_{0}, \varrho_{\mathrm{e}}, B_{0},|z|>a .\end{array}\right.$

Here the width of the slab, $2 a$, is chosen equal to $2.5 \mathrm{Mm}$ and the length of the slab is about $2 L=200 \mathrm{Mm}$. The initial configuration of the slab is shown in Fig. 1 (top panel). We take the mass density within the slab to be 3 times larger than in the ambient medium, $d=\varrho_{\mathrm{i}} / \varrho_{\mathrm{e}}=3$, following Aschwanden \& Nightingale (2005). Additionally, dense plasma layers are implemented at $0 \leq x \leq 0.05 L$ and $2.05 L \leq x \leq 2.1 L$ (Fig. 1, top panel). These represent the dense photospheric layer at the loop footpoints.

For comparison, $B_{0}$ is chosen to be equal to the average value of the magnetic field within the curved slab which we describe in the next part of the paper.

\subsection{An arcade model of a curved slab}

We adopt the coronal arcade model (e.g., Oliver et al. 1998) which was recently used by Gruszecki et al. (2006) in their studies of vertical oscillations of a multi-stranded loop.

We assume that in equilibrium the coronal arcade is settled in a motionless environment $\left(\boldsymbol{V}_{\mathrm{e}}=0\right)$. As a consequence of this approximation we use the current-free magnetic field condition $\left(\nabla \times \boldsymbol{B}_{\mathrm{e}} / \mu=0\right)$ which is satisfied by a magnetic potential $\boldsymbol{A}=A \hat{\boldsymbol{y}}$ :

$A(x, z)=B_{0} \Lambda_{\mathrm{B}} \cos \left(x / \Lambda_{\mathrm{B}}\right) \mathrm{e}^{-z / \Lambda_{\mathrm{B}}}$.

The components of the corresponding potential magnetic field are given by:

$B_{\mathrm{e} x}=B_{0} \cos \left(x / \Lambda_{\mathrm{B}}\right) \mathrm{e}^{-z / \Lambda_{\mathrm{B}}}$,

$B_{\mathrm{e} z}=-B_{0} \sin \left(x / \Lambda_{\mathrm{B}}\right) \mathrm{e}^{-z / \Lambda_{\mathrm{B}}}$.

Here $B_{0}$ is the magnetic field at the reference level $z=0, \Lambda_{\mathrm{B}}=$ $2 L / \pi$ is the magnetic scale height and $L$ is the horizontal halfwidth of the arcade, which we choose as $L=100 \mathrm{Mm}$.

From Eq. (2) we infer that for current-free magnetic field there is no pressure gradient, $\nabla p_{\mathrm{e}}=0$. As a result of that $p_{\mathrm{e}}=$ const. and mass density can be chosen as constant, $\varrho_{\mathrm{e}}=$ const.
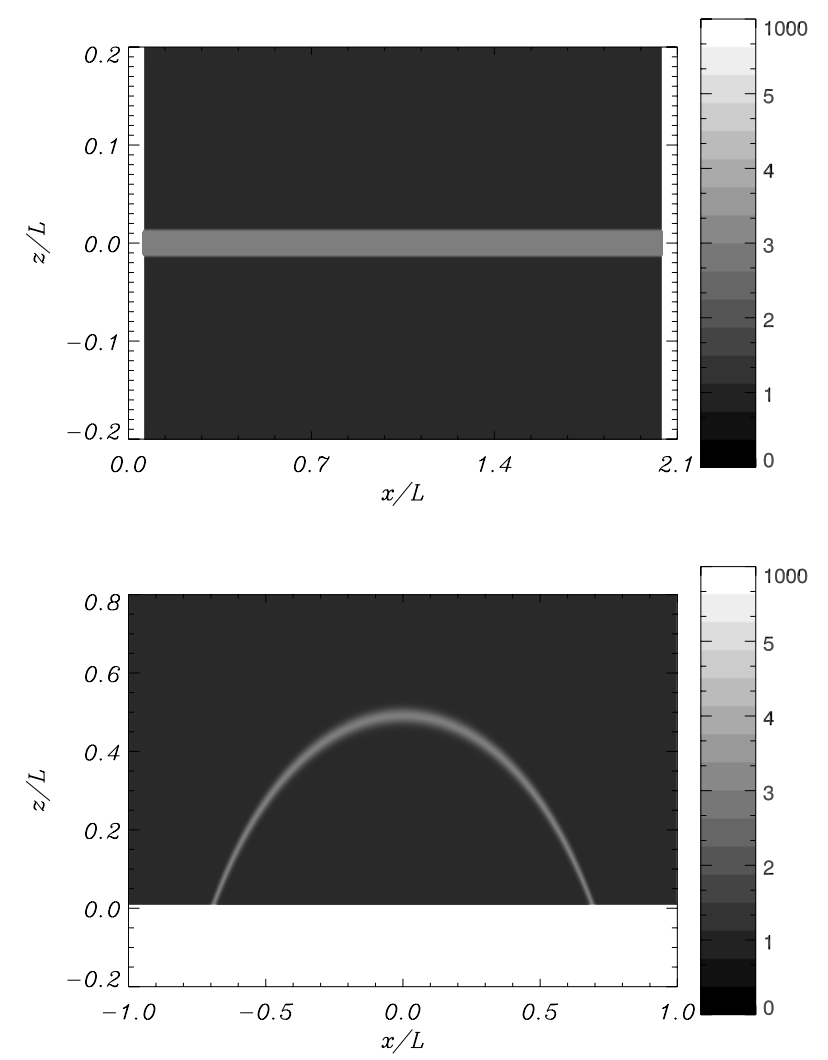

Fig. 1. Equilibrium mass density profiles representing straight (top panel) and curved (bottom panel) coronal slabs. Note the dense photospheric layers at $x \simeq 0$ and at $x \simeq 2 L$ (top panel) and at $z \simeq 0$ (bottom panel).

For this choice of the plasma parameters the Alfvén speed $V_{\mathrm{Ae}}(z)=\left|B_{\mathrm{e}}(z)\right| / \sqrt{\mu \varrho_{\mathrm{e}}}$ decays exponentially with height $z$. From the definition of the plasma beta, $\beta=p_{\mathrm{e}} /\left(B_{\mathrm{e}}^{2}(z=0) / 2 \mu\right)=$ $2\left(c_{\mathrm{S}} / V_{\mathrm{A}}(z=0)\right)^{2} / \gamma$, we can find the value of gas pressure. For typical coronal magnitudes of the sound and Alfvén speeds, $c_{\mathrm{s}}=300 \mathrm{~km} \mathrm{~s}^{-1}$ and $V_{\mathrm{A}}(z=0)=1000 \mathrm{~km} \mathrm{~s}^{-1}$, we obtain $\beta=0.108$ at the reference level $z=0$.

We set a curved slab by using the following mass density profile:

$\varrho(x, z)=\mathrm{d} \varrho_{\mathrm{e}} \exp \left(\frac{A(x, z)-A_{\mathrm{k}}}{2 a}\right)^{2}$,

where $A_{\mathrm{k}}=A\left(L_{\mathrm{f}}-a_{\mathrm{k}}, 0\right)$. We choose and hold fixed $2 a=0.02 L$, $L_{\mathrm{f}}=0.7 L$ and $a_{\mathrm{k}}=0.0125 L$. For this choice of the parameters the curved slab does not have ideal circular shape. The average radius and length of this slab are equal to $\sim 70 \mathrm{Mm}$ and $\sim 190 \mathrm{Mm}$, respectively.

We implement a dense photospheric layer (Fig. 1). This layer is modeled as

$\varrho(x, z)=\varrho_{\mathrm{e}}(\kappa-1)\left[1-\tan \left(\frac{z}{\sigma_{\mathrm{ph}}}\right)\right]$

where $\kappa=\varrho_{\mathrm{ph}} / \varrho_{\mathrm{e}}$ denotes mass density contrast of the photosphere and $\sigma_{\mathrm{ph}}$ is a steepness of the mass density along the $z$-direction at the photosphere. We choose $\sigma_{\mathrm{ph}}=0.0025 \mathrm{~L}$ and $\varrho_{\mathrm{ph}}=1000 \varrho_{\mathrm{e}}$. Such a dense plasma layer results in wave reflection. 


\subsubsection{Perturbations}

We are interested in the impulsively excited Alfvén waves. For the straight slab we launch the initial pulse in $V_{y}$, i.e.

$V_{y}(x, z, t=0)= \begin{cases}V_{0} \mathrm{e}^{-\left(x-x_{0}\right)^{2} / w^{2}}, & |z| \leq a, \\ 0, & |z|>a,\end{cases}$

where $x_{0}$ denotes the pulse's initial position, $w$ is its width and $V_{0}$ is its amplitude that is expressed in units of the Alfvén speed, $V_{\mathrm{A}}$. We set the width of the initial pulse, $w=50 \mathrm{Mm}$. For this choice of the parameters the pulse is placed exactly in the slab center, at $x_{0}=L$.

For the curved slab we launch a pulse in $V_{y}$. We choose a pulse that is confined to the curved slab, similar to the straight slab case.

\section{Numerical results}

To obtain numerical results we use the code ATHENA. This code was developed by Gardiner \& Stone (2005). Athena is a gridbased code for astrophysical plasma dynamics applications. The numerical algorithm in the code is based on a higher-order Godunov method with a single-step Eulerian update. Numerical fluxes are computed using a linearized Riemann solver and the divergence-free condition is satisfied with the use of a constraint transport method. This code can be adopted to solve the equations of compressible hydrodynamics and ideal MHD problems in one or two spatial dimensions. In our studies we use the code for ideal MHD equations in 2D space. To represent a physical region we use a Eulerian box $(0,2.1 L) \times(-L, L)$ $((-L, L) \times(-0.2 L, 1.3 L))$ in the case of the straight (curved) slab. This box is covered by $500 \times 500$ grid points. We set transparent boundary conditions at all boundaries of the simulation region allowing a wave signal to freely leave the simulation area. We perform convergence studies in order to check the influence of grid size on numerical results. We choose a finer grid and compare results for two different grids. From the obtained results we infer that numerical results are not affected by numerical diffusion and they are well represented by the chosen grid.

\subsection{The straight slab}

We discuss now the case of the straight slab. First we perform a simulation for an infinitely dense photospheric layer for which $\varrho_{\text {ph }} \rightarrow \infty$. In practise, this case is realized by removing the photospheric layers from the system and implementing reflecting boundary conditions at $x=0$ and $x=2 L$.

As our goal is to study linear waves first we launch a pulse with a small amplitude, $V_{0}=0.01 V_{\mathrm{A}}$. Figure 2 sketches the temporal behaviour of $V_{y}$ at a spatial point $x / L=1$ and $z / L=0$. Alfvén wave signal is essentially constant in time. A more detailed analysis can be done using Alfvén wave energy, which consists of kinetic and magnetic energies, viz.

$E_{\mathrm{Al}}(t)=\int_{S} \int\left(\frac{\varrho V_{y}^{2}}{2}+\frac{B_{y}^{2}}{2 \mu}\right) \mathrm{d} x \mathrm{~d} z$.

Here the integration is performed over the slab area $S$. As $E_{\mathrm{Al}}$ decays in time it is discernible from Fig. 3 that the Alfvén wave experiences attenuation due to lateral leakage into the ambient coronal medium. However, as at $t=10000 \mathrm{~s}$ (corresponding to 11 wave periods, where the wave period is defined as $P=$ $2 L / V_{\mathrm{A}}$ ) the Alfvén wave has lost only $0.73 \%$ of its initial energy;

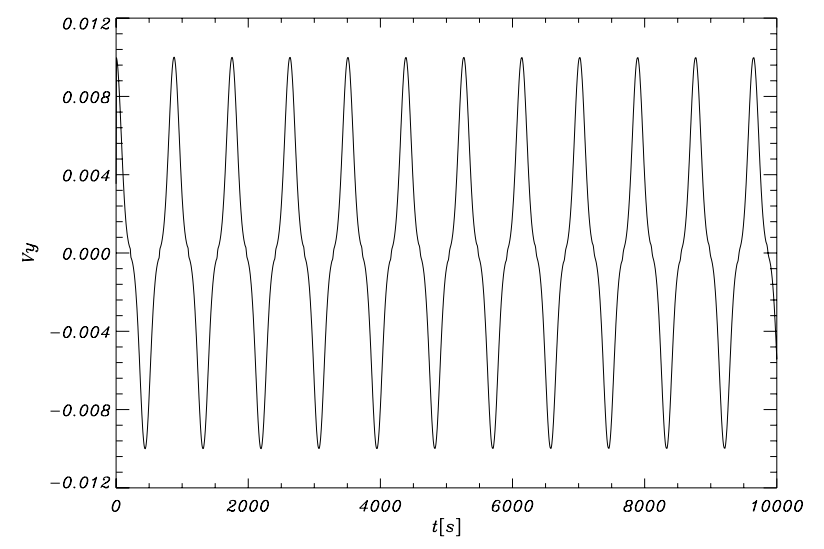

Fig. 2. Time signature of the Alfvén wave signal, collected at $z / L=0$, for the case of $\varrho_{\mathrm{ph}} \rightarrow \infty$ and $V_{0}=0.01 V_{\mathrm{A}}$.

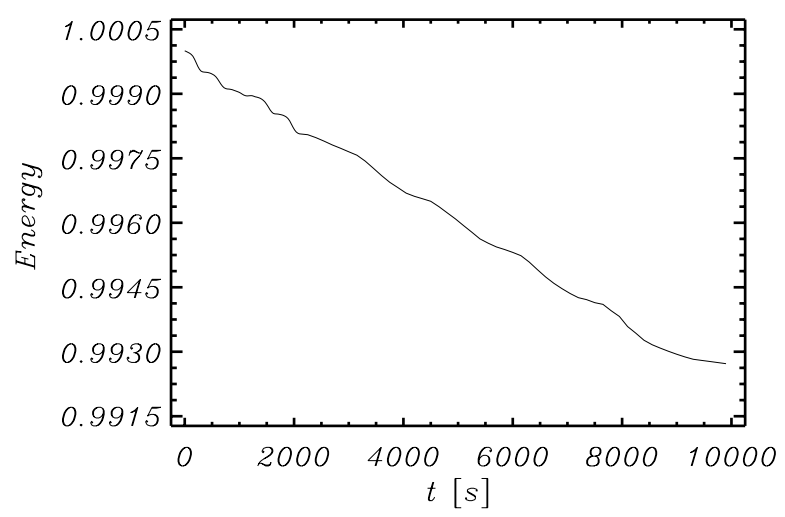

Fig. 3. Time-signatures of the normalized Alfvén wave energy, $E_{\mathrm{Al}}(t) / E_{\mathrm{Al}}(0)$, within the slab for the case of Fig. 2.

we infer that the lateral leakage of Alfvén waves is negligibly small. It is noteworthy that the Alfvén wave gradually loses its energy and there is no initial transient phase at which the energy decreases significantly. Such a phase was observed in the case of fast sausage (Pascoe et al. 2007) and slow (Ogrodowczyk et al. 2006) magnetosonic oscillations.

We consider now different values of the amplitude of the initial pulse, $V_{0}$. Figure 4 shows the temporal evolution of energy loss for: $V_{0}=0.01 V_{\mathrm{A}}$ (crosses), $V_{0}=0.05 V_{\mathrm{A}}$ (stars), $V_{0}=0.1 V_{\mathrm{A}}$ (triangles) and $V_{0}=0.2 V_{\mathrm{A}}$ (squares). Here the energy loss, $E_{\mathrm{loss}}$, is defined as:

$E_{\mathrm{loss}}=\frac{E_{\mathrm{Al}}(t=0)-E_{\mathrm{Al}}(t)}{E_{\mathrm{Al}}(t=0)} \cdot 100 \%$.

From Fig. 4 we infer that for a larger value of $V_{0}$ the Alfvén wave loses energy at a higher absolute and fractional rate. This scenario is in agreement with our expectation that for larger $V_{0}$ the Alfvén waves become more nonlinear and drive higher amplitude magnetosonic waves (Nakariakov et al. 1997) and as a consequence of that lose more energy (Murawski 1992).

We evaluate now the attenuation time, $\tau$, for four different values of the amplitude. Here, $\tau$ is determined by fitting the following formula:

$V_{y}(t)=V_{y}(t=0) \exp (-t / \tau)$

to the corresponding wave signal. The results are displayed in Fig. 5. We see that $\tau / P$ declines rapidly with $V_{0}$. Here $P$ is the wave period. It means that a larger amplitude Alfvén wave loses a given fraction of its energy faster than a pulse with 


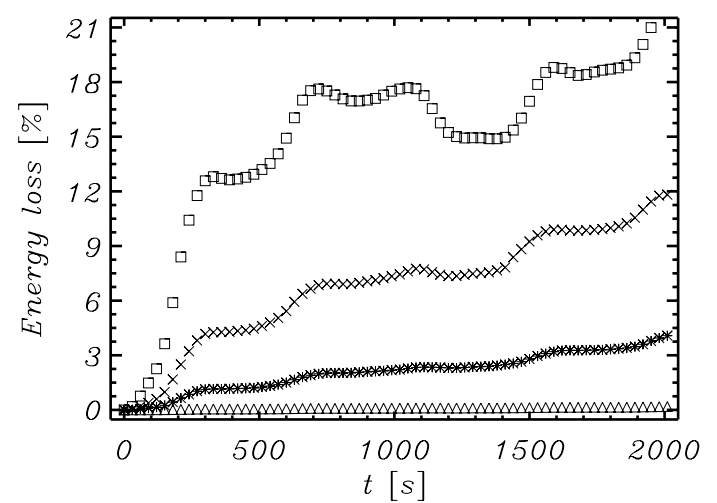

Fig. 4. Energy loss of Alfvén waves within the slab for four different amplitudes of the initial pulse: $V_{0}=0.01$ (triangles), $V_{0}=0.05$ (stars), $V_{0}=0.1$ (crosses) and $V_{0}=0.2$ (squares) in units of $V_{\mathrm{A}}$.

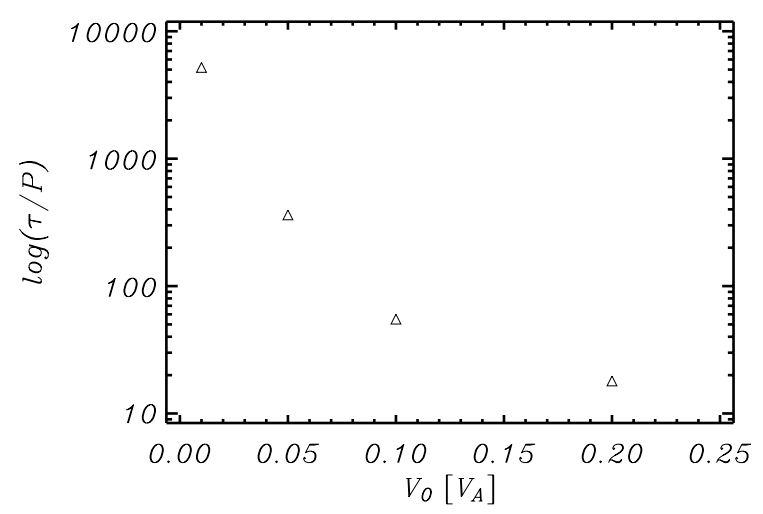

Fig. 5. The ratio of the attenuation time to the wave period, $\tau / P$, vs. amplitude of the initial pulse, $V_{0}$.

smaller $V_{0}$. Figure 6 shows the profiles of $V_{x}$ for two different values of $V_{0}$. It is clearly visible that for $V_{0}=0.2 V_{\mathrm{A}}, V_{x}$ attains higher magnitudes than in the case of $V_{0}=0.01 V_{\mathrm{A}}$. This serves as evidence that higher amplitude Alfvén waves efficiently drive higher amplitude magnetosonic waves. As a result of this process Alfvén waves lose their energy faster for larger values of $V_{0}$.

Up to now the photospheric layers have been removed from the system and their action was replaced by reflecting boundary conditions. Figure 7 shows energy loss of the Alfvén waves, $E_{\text {loss }}$, within the slab for the straight slab case with the photospheric layers present in the system. We launch a pulse with $V_{0}=0.01 V_{\mathrm{A}}$. At $t=2000 \mathrm{~s}$ the Alfvén wave lost almost $70 \%$ of its initial energy. A comparison with Fig. 4 suggests that this energy loss results from energy leakage into the photospheric layers. The case when the Alfvén speed jumps discontinuously from the photospheric to the coronal value, the reflection coefficient can be approximated as (Ferraro 1950)

$R=\frac{V_{\mathrm{As}}-V_{\mathrm{Ap}}}{V_{\mathrm{As}}+V_{\mathrm{Ap}}}$

For example for the case $\varrho_{\mathrm{ph}}=1000 \varrho_{\mathrm{c}}$, where $\varrho_{\mathrm{c}}$ denotes the density of the corona, $V_{\mathrm{As}}=456 \mathrm{~km} \mathrm{~s}^{-1}\left(V_{\mathrm{Ap}}=25 \mathrm{~km} \mathrm{~s}^{-1}\right)$ is the Alfvén speed in the slab (photosphere). For these values we get $R=0.896$ which means that at every reflection about $10 \%$ of Alfvén wave energy is lost due to energy leakage into a single photospheric layer. As wave reflections occur at both slab ends the total amount of energy, which is lost during a partial reflection, is about $20 \%$. Indeed, Fig. 7 (triangles) shows that at the first reflection, which takes a place at $t=300 \mathrm{~s}$, the Alfvén wave
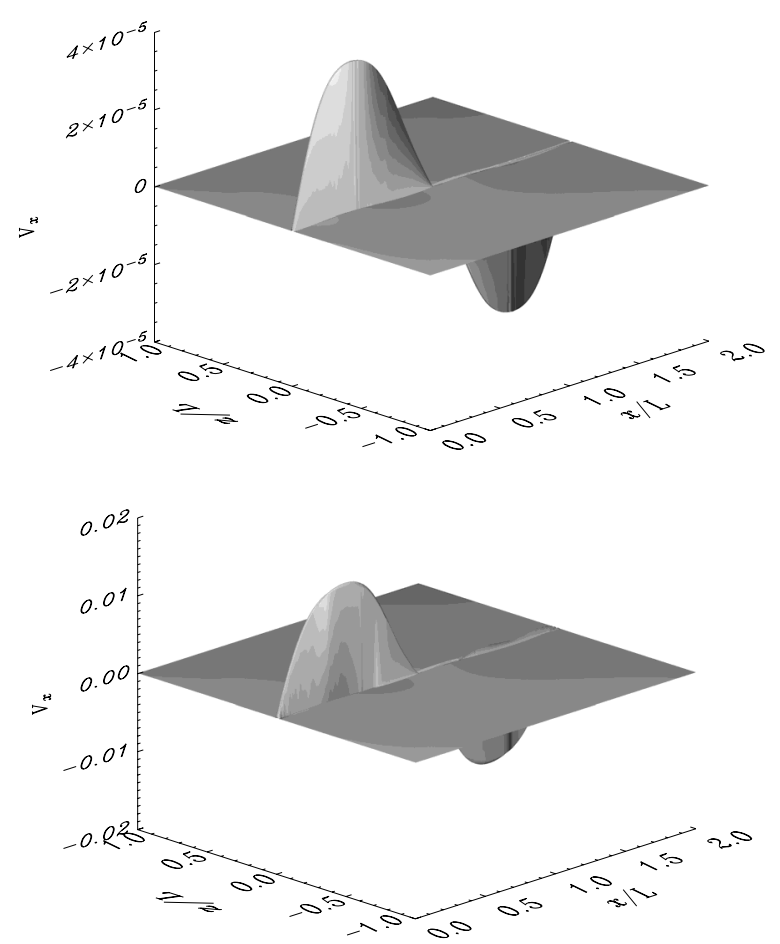

Fig. 6. Spatial profiles of $V_{x}$ at $t=150 \mathrm{~s}$ for $V_{0}=0.01 V_{\mathrm{A}}$ (first panel) and $V_{0}=0.2 V_{\mathrm{A}}$ (second panel).

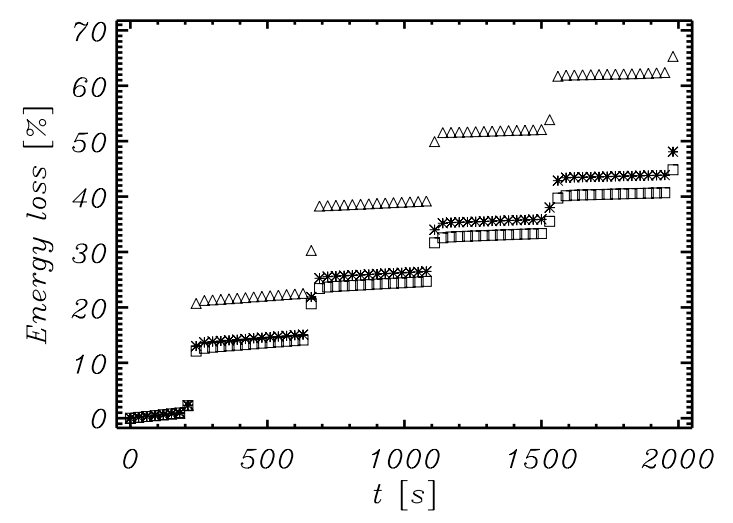

Fig. 7. Energy loss of Alfvén waves within the slab with the photospheric layers for $V_{0}=0.01$ and the width of the pulse $w=5 \mathrm{Mm}$. We estimate energy loss for three different values of the photospheric mass density: $\varrho_{\mathrm{ph}}=1000 \varrho_{\mathrm{c}}$ (triangles), $\varrho_{\mathrm{ph}}=5000 \varrho_{\mathrm{c}}($ stars $), \varrho_{\mathrm{ph}}=10000 \varrho_{\mathrm{c}}$ (squares).

lost about $20 \%$ of its initial energy. We also estimate values of $R$ for the cases $\varrho_{\mathrm{ph}}=5000 \varrho_{\mathrm{c}}(R=0.95)$ and $\varrho_{\mathrm{ph}}=10000 \varrho_{\mathrm{c}}$ $(R=0.96)$, which agree with the result of Fig. 7. From Eq. (15) we also estimate a value of $R$ for the realistic case $\varrho_{\mathrm{ph}}=10^{9} \varrho_{\mathrm{c}}$. We get $R=0.99989$. As a result we infer that energy loss at the photospheric regions is much less significant than lateral leakage and leakage due to nonlinear driving of magnetosonic waves for the real value of density gradient between photosphere and corona.

\subsection{The curved slab}

We now consider the curved slab model of an arcade loop. Figure 8 shows energy loss of Alfvén waves within the curved slab for $V_{0}=0.01 V_{\mathrm{A}}$. Similarities between straight and curved slabs are discernible (compare Figs. 8 and 7). Note that the 


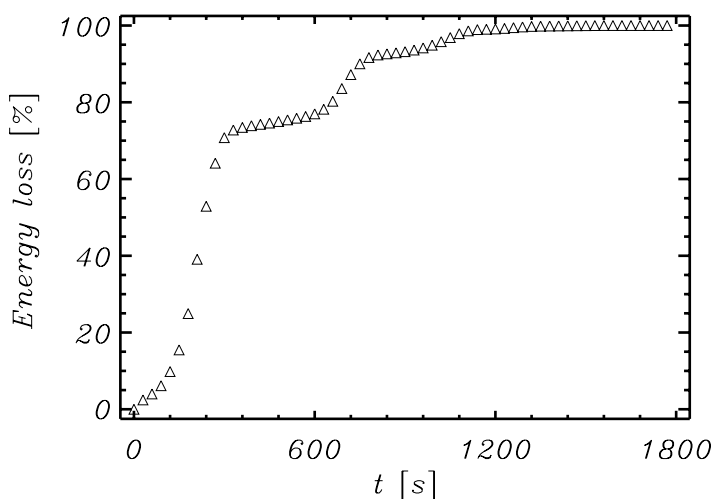

Fig. 8. Energy loss of the Alfvén waves within the curved slab for $V_{0}=$ $0.01 V_{\mathrm{A}}$.

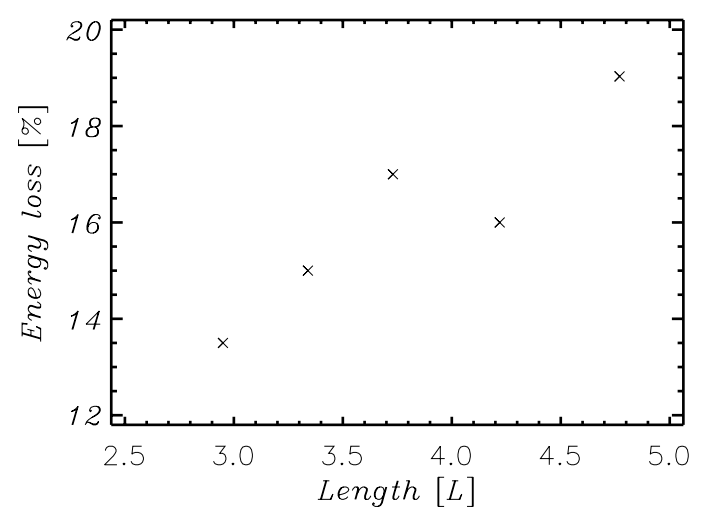

Fig. 9. Relative energy loss of Alfvén waves within the curved slab for different slab lengths.

Alfvén wave loses its energy while being partially reflected from the photospheric region. The first three reflections occur at $t \simeq 300 \mathrm{~s}, t \simeq 700 \mathrm{~s}, t \simeq 1100 \mathrm{~s}$. At the intermediate times, when the Alfvén wave propagates along the curved slab, energy decreases at a significantly lower rate. This energy loss results from energy leakage into the ambient corona as a result of the curvature of magnetic field lines. To analyze this effect we evaluate energy loss for five different lengths of the curved slab. The corresponding results are displayed in Fig. 9. Note that for a longer slab, magnetic field lines are bent more than for a shorter slab. As a consequence we expect that curvature effects are stronger for longer slabs. Indeed, we see in Fig. 9 that energy loss of Alfvén waves, $E_{\text {loss }}$, within the curved slab grows with the length of the slab. Thus, Alfvén waves that propagate along the slab of higher curvature lose energy at a higher rate than Alfvén waves in less curved slabs.

\section{Summary and conclusions}

In this paper, attenuation of Alfvén waves in straight and curved coronal slabs is studied in a $2.5 \mathrm{D}$ Cartesian geometry. In the present simulation the Alfvén waves are triggered by a sudden energy release within a slab in the form of a velocity pulse and then the system is allowed to evolve in time. As a result of energy leakage Alfvén waves decay in time. For small amplitude (linear) Alfvén waves the decay rate is negligibly small due to lateral leakage as it reaches a level of 3\% energy loss after 10000 s. Larger amplitude Alfvén waves excite the fast magnetosonic waves nonlinearly, and show stronger damping. For realistic values of photospheric and coronal density ratios the energy leakage rate is small compared to lateral losses. We find that wave attenuation is enhanced by about a factor of 2 by curvature effects and it is stronger for larger amplitude Alfvén waves, which are able to drive magnetosonic waves more efficiently than small amplitude Alfvén waves.

Comparing the $1.5 \mathrm{D}$ case that was discussed by Ofman (2002) with the 2.5D simulations of the present study, we infer that lateral leakage of Alfvén waves, that is absent in the $1.5 \mathrm{D}$ case, provides another mechanism for Alfvén wave dissipation. However, the $1.5 \mathrm{D}$ model describes sufficiently well the attenuation of Alfvén waves by photospheric losses.

Acknowledgements. L.O. would like to thank the NASA SR\&T program and the NASA Sun-Earth Connection Theory Program for support. Athena is a gridbased code for astrophysical gas dynamics being developed with support of the NSF Information Technology Research (ITR) program. The magnetohydrodynamics code used in this study was developed at Princeton University by Tom Gardiner, Jim Stone, Peter Teuben and John Hawley with support of the NSF Information Technology Research program.

\section{References}

Aschwanden, M. J., \& Nightingale, R. W. 2005, ApJ, 633, 499

Aschwanden, M. J., Fletcher, L., Schrijver, C. J., \& Alexander, D. 1999, ApJ, 520,880

Aschwanden, M. J., De Pontieu, B., Schrijver, C. J., \& Title, A. M. 2002, Sol. Phys., 206, 99

Cally, P. S. 1986, Solar Phys., 103, 277

Del Zanna, L., Schaekens, E., \& Velli, M. 2005, A\&A, 431, 1095

De Moortel, I., Ireland, J., Walsh, R. W., \& Hood, A. W. 2002, Sol. Phys., 209, 61

Edwin, P. M., \& Roberts, B. 1982, Sol. Phys., 76, 239

Edwin, P. M., \& Roberts, B. 1983, Sol. Phys., 88, 179

Ferraro, V. C. A. 1950, ApJ, 119, 393

Gardiner, T. A., \& Stone, J. M. 2005, J. Comput. Phys., 205, 509

Gruszecki, M., Murawski, K., Selwa, M., \& Ofman, L. 2006, A\&A, 460, 887

Mendoza-Briceno, C. A., Erdélyi, R., Sigalotti, L., \& Di, G. 2004, ApJ, 605, 493

Murawski, K. 1992, Solar Phys., 139, 279

Murawski, K., \& Roberts, B. 1994, Solar Phys., 65, 75

Nakariakov, V. M. 2003, in The Dynamic Sun, ed. B. Dwivedi, CUP, 314

Nakariakov, V. M., Roberts, B., \& Murawski, K. 1997, Solar Phys., 175, 93

Nakariakov, V. M., Ofman, L., Deluca, E. E., Roberts, B., \& Davila, J. M. 1999, Science, 285,862

Nakariakov, V. M., Melnikov, V. F., \& Reznikova, V. E. 2004, IAU Symp., 653

Ofman, L. 2002, ApJ, 568, L135

Ogrodowczyk, R., Murawski, K., Solanki, S. K., \& Nakariakov, V. M. 2006, $\mathrm{A} \& \mathrm{~A}$, in preparation

Oliver, R., Murawski, K., \& Ballester, J. L. 1998, A\&A, 330, 726

Pascoe, D. J., Nakariakov, V. M., \& Arber, T. D. 2007, A\&A, 461, 1149

Roberts, B. 2000, Sol. Phys., 193, 139

Roberts, B., Edwin, P. M., \& Benz, A. O. 1984, ApJ, 279, 857

Schrijver, C. J., Aschwanden, M. J., \& Title, A. M. 2002, Sol. Phys., 206, 69

Selwa, M., Murawski, K., Kowal, G., et al. 2004, A\&A, 495

Selwa, M., Murawski, K., Solanki, S. K., Wang, T. J., \& Tóth, G. 2005, A\&A, 440,385

Terradas, J., Oliver, R., \& Ballester, J. L. 2006, ApJ, 650, L91

Wang, T. J., \& Solanki, S. K. 2004, A\&A, 421, L33

Wang, T. J., Solanki, S. K., Curdt, W., Innes, D. E., \& Dammasch, I. E. 2002, 199

Van Doorsselaere, T., Debosscher, A., Andries, J., \& Poedts, S. 2004, A\&A, 424, 1065

Zaqarashvili, T. V. 2003, A\&A, 399, L15 